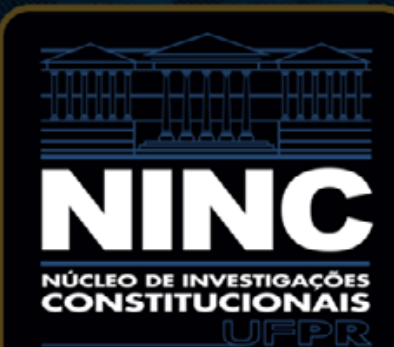




\title{
La dynamique de la déférence: création et évolution des modèles auto-restrictives de contrôle juridictionnel dans le droit comparé*
}

\section{The dynamics of deference: the creation and the evolution of deferential standards of judicial review in comparative law}

\author{
EDUARDO JORDÃO** \\ Fundação Getúlio Vargas - FGV DIREITO RIO (Brasil) \\ eduardo.jordao@fgv.br
}

Recebido/Received: 25.06 .2015 / June 25th, 2015

Aprovado/Approved: 27.07.2015 / July 27th, 2015

\section{Résumé}

Cet article examine la création et l'évolution des modèles déférentiels de contrôle dans quatre systèmes juridiques (France, Italie, Etats-Unis et Canada). Cette perspective historique servira à démontrer (i) que la position juridictionnelle auto-restrictive peut avoir des significations très distinctes et (ii) que dans certains systèmes juridiques, la déférence est fortement disséminée, alors que dans d'autres, elle se trouve en plein déclin.

Mots-clés: dynamique de la déférence; contrôle juridictionnel; administration publique; création et évolution droit comparé.

\section{Abstract}

This paper examines the creation and the evolution of standards of review in four jurisdictions (France, Italy, United States and Canada). This historical perspective will demonstrate (i) that the judicial self-restraint can have different meanings; and (ii) that while in certain jurisdictions the deferential standards of review are disseminated, in others they are experiencing a clear decline.

Keywords: dynamics of deference; judicial review; public administration; creation and evolution; comparative law.

Como citar esse artigo/How to cite this article: JORDÃO, Eduardo. La dynamique de la déférence: création et évolution des modèles auto-restrictives de contrôle juridictionnel dans le droit comparé. Revista de Investigações Constitucionais, Curitiba, vol. 2, n. 3, p. 111-135, set./dez. 2015. DOI: http://dx.doi.org/10.5380/rinc.v2i3.44531

* Cet article est une version adaptée d'une section du dernier chapitre de la thèse de l'auteur. La thèse a obtenu le Prix Levy-UIIman de la Chancellerie des Universités de Paris.

** Professeur à l'Ecole de Droit de Rio de Janeiro de la Fundação Getulio Vargas - FGV Direito Rio (Rio de Janeiro-RJ, Brasil). Docteur en Droit Public par l'Université Paris 1 (Panthéon-Sorbonne) et I'Università di Roma (Sapienza), en cotutelle. 


\section{SOMMAIRE}

1. Les circonstances historiques de la création des modèles déférents; 1.1. L'hypothèse intuitive: le modèle déférent comme instrument de rétraction juridictionnelle; 1.1.1. L'illustration parfaite du droit canadien; 1.1.2. Les illustrations moins claires des droits américain et italien; 1.1.2.1. Le droit américain et la doctrine Chevron; 1.1.2.2. Le « contrôle faible » des évaluations techniques complexes en Italie; 1.2. L'hypothèse contre-intuitive: le modèle déférent pour l'élargissement de l'intervention; 1.2.1. La création du modèle de contrôle restreint en 1961 en France; 2. L'évolution historique du modèle de contrôle déférent; 2.1. L'hypothèse de dissémination du modèle déférent; 2.1.1. L'illustration claire du droit canadien: le déclin du modèle de la correction; 2.1.2. L'illustration moins claire du droit américain: le mouvement pendulaire; 2.2. L'hypothèse de déclin ou d'abandon du modèle déférent; 2.2 .1 . L'abandon total du contrôle faible en droit italien; 2.2.2. Le modèle de contrôle restreint français comme « rituel de passage »; 3 . Références.

De nombreuses études comparent l'intensité du contrôle des juges dedifférentes nationalités sur les décisions de l'administration publique. ${ }^{1}$ Les donnés produits permettent d'identifier une variation dans le degré du « déférence »2juridictionnel aux décisions administratives, en révélant des orientations plus ou moins interventionnistes des juges d'un pays donné, face à ses collègues étrangers.

Le fait est que la majorité de ces études adopte une perspective statique : elles révèlent des différences actuelles, courantes des orientations jurisprudentielles. Cet article propose une perspective différente pour affronter la même problématique : l'examen de la dynamique de l'intensité du contrôle juridictionnel, par le biais d'une approche historique sur la création et l'évolution des modèles déférentiels de contrôle de quatre systèmes juridiques(France, Italie, Etats-Unis et Canada). La perspective historique ou dynamique permet une compréhension plus complète (et plus fiable) de

\footnotetext{
1 V., par exemple, ESSENS, Oda; GERBRANDY, Anna; LAVRIJSSEN, Saskia (Ed.). National Courts and the Standard of Review in Competition Law and Economic Regulation. Groningen: Europa Law Publishing, 2009; LI, Zhang. Le Contrôle Juridictionnel de la Légalité des Actes Administratifs en Chine: Eléments d'Analyse Comparée des Contentieux Administratifs Chinois et Français. Bruxelles: Bruylant, 2010; BONNARD, Roger. Le Contrôle Juridictionnel de l'Administration: Étude de Droit Administratif Comparé. Paris: Dalloz, 2005; MARCHETTI, Barbara. Pubblica Amministrazione e Corti negli Stati Uniti: il Judicial Review sulle Administrative Agencies. Padova: CEDAM, 2005.

2 Dans ce travail, le terme « déférence » dénote une attitude ou un positionnement respectueux, une orientation auto-restrictive de juges ou juges spécifiques. Dans ce sens, affirmer qu'un juge a appliqué la « déférence » dans le contrôle juridictionnel d'une décision de l'administration publique signifie que le juge a considéré la solution administrative avec du respect. Plus précisément, cela signifie que le juge n'a pas considéré directement la question juridique (ou, par exemple, qu'il n'a pas interprété directement la législation en cause), mais qu'il a considéré uniquement le caractère raisonnable, cohérent, logique, de la décision ou interprétation adoptée par l'autorité administrative. Cela se produit (ou du moins devrait se produite) lorsque les juges appliquent des modèles déférents de contrôle juridictionnel, comme celui du caractère raisonnable au Canada, ou le contrôle faible en Italie, le contrôle restreint en France, ou la doctrine Chevron aux Etats Unis. Dans le sens ici déployé, la déférence n'implique pas nécessairement le maintien de la décision administrative: en utilisant n'importe quel des modèles énumérés ci-dessus, il est possible que le juge conclue au final que la décision administrative $n^{\prime} a$ pas été, par exemple, raisonnable, ou permissible, ou cohérent, ou logique - et qu'il décide de l'annuler. Même dans ces cas, il y aurait eu de la déférence juridictionnelle, du moins selon ce sens d'« attitude respectueuse ».
} 
l'orientation adopté par chaque pays en ce qui concerne la relation entre le juge et les autorités administratives.

D'un côté, l'attention à l'évolution jurisprudentielle, surtout dans les droits canadiens et français, laisse transparaître que la position juridictionnelle auto-restrictive peut avoir des significations historiques très distinctes. La création d'un modèle de contrôle déférent ne sert pas toujours à exprimer un mouvement de rétraction juridictionnelle ; elle peut aussi (contra-intuitivement) servir à rendre viable un élargissement de l'intervention juridictionnelle, dans des domaines qui ne relevaient d'aucun contrôle auparavant.

D'un autre côté, la perspective dynamique accentue la façon par laquelle le modèle de contrôle déférent a fait l'objet d'évolutions différentes dans les systèmes juridiques étudiés : dans certains, il a été fortement disséminé, au point de constituer le modèle de contrôle juridictionnel « standard »; dans d'autres, il se trouve en plein déclin, en laissant de la place au contrôle juridictionnel non déférent.

L'article est consacré à ces deux analyses. En premier lieu, nous traiterons des raisons historico-jurisprudentielles de la création du modèle de contrôle juridictionnel déférent (1). En second lieu, nous nous pencherons sur l'analyse de l'évolution du modèle (2).

\section{LES CIRCONSTANCES HISTORIQUES DE LA CRÉATION DES MO- DĖLES DÉFÉRENTS}

Tout d'abord, il convient d'exposer les circonstances qui, dans chaque juridiction, ont conduit à la création d'un modèle de contrôle déférent. Dans I'hypothèse la plus intuitive, ce modèle de contrôle implique un mouvement de rétraction juridictionnelle. Dans ce sens, sa création permettrait de restreindre un contrôle juridictionnel qui était trop intense auparavant (1.1). Mais il existe une seconde hypothèse, moins évidente: celle selon laquelle la création d'un modèle de contrôle juridictionnel déférent sert à permettre l'élargissement de l'intervention juridictionnelle, dans des domaines qui n'étaient soumis à aucun contrôle auparavant (1.2).

\subsection{L'hypothèse intuitive: le modèle déférent comme instrument de rétraction juridictionnelle}

L'hypothèse intuitive trouve une illustration très claire au Canada (1.1.1) et des illustrations un peu moins évidentes aux États Unis et en Italie (1.1.2).

\subsubsection{L'illustration parfaite du droit canadien}

En droit canadien, la création du premier modèle déférent de contrôle (le modèle du caractère manifestement déraisonnable) traduit un mouvement évident de 
rétraction juridictionnelle ; elle implique un développement dans la direction d'un contrôle moins intense que celui qui avait lieu précédemment. Ce modèle de contrôle a été créé sous l'affaire CUPE, en 19793. L'initiative correspond à une réaction de la Cour suprême vis-à-vis d'une attitude progressivement interventionniste des organes juridictionnels inférieurs ${ }^{4}$.

La situation avait conduit à une claire insatisfaction de l'administration publique et du Parlement. En guise de réponse, le Parlement avait renforcé la promulgation de législations contenant les dénommées « clauses privatives », qui sont des dispositions tendant à restreindre l'intervention juridictionnelle. Ces clauses établissaient, par exemple, que les décisions d'une autorité administrative donnée étaient « finales et conclusives » ou les excluaient de façon directe et explicite du contrôle juridictionnel. Mais cette intervention du Parlement n'avait pas engendré l'effet souhaité. Les juges se sont mis à contourner cet obstacle par le biais de l'interprétation selon laquelle les clauses privatives ne couvraient pas des « questions juridictionnelles », lesquelles seraient toujours soumises à un contrôle juridictionnel non déférent ${ }^{5}$. Par la suite, les questions juridictionnelles ont été interprétées de manière large, servant de fondement théorique dont le mouvement juridictionnel avait besoin.

Ainsi, la situation d'un haut interventionnisme a subsisté, accompagnée également de l'insatisfaction des pouvoirs exécutif et législatif.6 Dans l'affaire CUPE, la Cour

\footnotetext{
3 Canadian Union of Public Employees (CUPE), Local 963 v. New Brunswick (Liquor Corp.), [1979] 2 SCR 227

4 MULLAN, David J. Recent developments in administrative law - The apparent triumph of deference! Canadian Journal of Administrative Law and Practice, v. 12, n. 2, p. 191-, 1998. p. 192.
}

5 LANGILLE, Brian. Judicial review, judicial revisionism and judicial responsibility. Revue Générale de Droit, Ottawa, v. 17, n. 1-2, p. 169-192, 1986. p.189-190: «Insofar as a privative clause stood in the way of the Court's inflicting their own views upon the parties the " comforting conceptualism of jurisdiction » offered a right of passage into forbidden territory. (...) What is crucial is that the bottom line was a major invasion by the courts of the decisionmaking processes established by national labour law policy, and the thwarting of those processes and of that policy as a result. Thus we became witnesses to a familiar and recurring bit of institutional choreography - a labour board or arbitration decision - judicial review quashing the decision - legislative overruling of the judges ».

6 D'un point de vue théorique, la période d'hostilité juridictionnelle vis-à-vis de l'administration publique était liée à une forte influence de l'individualisme et de la méfiance vis-à-vis de l'État administratif. V. J. WILLIS, John. Three approaches to administrative law: the judicial, the conceptual and the functional. The University of Toronto Law Journal, Toronto, v. 1, p. 53-81, 1935. p. 60; RISK, Richard C. B. In Memoriam: John Willis. The University of Toronto Law Journal, Toronto, v. 47, p. 301-304, 1997. p. 303; et DYZENHAUS, David. The logic of the rule of law: lessons from Willis. The University of Toronto Law Journal, Toronto, v. 55, n. 3, p. 691-714, jun./ sept. 2005. p. 691. Ces tendances ont significativement pris corps dans la doctrine de Albert Venn Dicey, professeur de I'Université d'Oxford et ardent opposant de l'existence d'un « droit administratif ». L'objection de DiCEY au droit administrative et sa phrase classique sont bien connues: «In England we know nothing of administrative law, and wish to know nothing about it ». Pour cet auteur, l'administration publique devait être soumise au même droit qui régissait l'action des personnes privées. La soumission à un droit spécial serait une forme injustifiée de privilège. Dans une affaire, le juge Wilson de la Cour suprême du Canada a clasiffié Dicey comme « remarkably influential » en droit administratif canadien (v. American Farm Bureau Federation v. Canadian Import Tribunal (sub nom. National Corn Growers Association v. Canada (Import Tribunal)), [1990] 2 S.C.R. 1324 at 1332). Dicey concentrait entre les mains du Pouvoir Judiciaire le rôle de grand défenseur de l'État de droit et proclamait la pertinence d'une forte intervention juridictionnelle afin d'éviter des arbitrarités administratives. 
suprême a décidé de mettre une fin à une telle situation, en annonçant et en inaugurant une ère nouvelle reposant sur davantage de restrictions pour les juges. Bien entendu, dans ces décisions précédentes, la Cour suprême avait déjà suggéré que l'auto restriction juridictionnelle pourrait être préférable7. Mais, dans ce cas, cela est fait de manière explicite pour la première fois. II y a un extrait célèbre du juge Dickson affirmant que les juges ne doivent pas chercher à faire passer comme étant juridictionnelles des questions qui le sont de manière incertaine, dans le seul but d'exercer un contrôle plus intense ${ }^{8}$. Il s'agit d'une condamnation explicite des manœuvres qui étaient faites par les juridictions inférieures afin d'attirer un contrôle plus intense.

Le jugement en soi est un libellé en faveur de la déférence juridictionnelle à l'administration publique. La Cour suprême fait d'importantes considérations sur la manière dont la procédure décisionnelle des agences diffère de la procédure décisionnelle des organes juridictionnels, en comprenant notamment la mise en balance de différents intérêts publics conflictuels ${ }^{9}$. Elle souligne encore la façon par laquelle les décisions des agences comprennent souvent des choix également raisonnables, face à l'ambigüité de la rédaction de la norme ${ }^{10}$. Étant donné cette ambigüité et la possibilité d'en dégager différentes solutions raisonnables, la question pertinente devient celle de savoir quelle est l'institution la mieux adaptée pour effectuer des tels choix.

L'affaire CUPE avait trait à l'interprétation d'une disposition de la législation du travail contrôlée par une agence administrative, relevant du « cœur » de sa compétence. Pour cette raison, conformément à la Cour suprême, l'agence avait même le «

\footnotetext{
7 Service Employees, International Union, Local 333 v. Nipawin District Staff Nurses' Assn., [1975] 1 SCR 382 ; Jacmainv.Canada (Attorney General), [1978] 2 SCR 15.

8 Canadian Union of Public Employees (CUPE), Local 963 v. New Brunswick (Liquor Corp.), [1979] 2 SCR 227 at 233: « With respect, I do not think that the language of « preliminary or collateral matter » assists in the inquiry into the Board's jurisdiction. One can, I suppose, in most circumstances subdivide the matter before an administrative tribunal into a series of tasks or questions and, without too much difficulty, characterize one of those questions as a « preliminary or collateral matter ». [...] The question of what is and is not jurisdictional is often very difficult to determine. The courts, in my view, should not be alert to brand as jurisdictional, and therefore subject to broader curial review, that which may be doubtfully so ».

9 Canadian Union of Public Employees (CUPE), Local 963 v. New Brunswick (Liquor Corp.), [1979] 2 SCR 227 at 235-236: «The usual reasons for judicial restraint upon review of labour board decisions are only reinforced in a case such as the one at bar. Not only has the Legislature confided certain decisions to an administrative board, but to a separate and distinct Public Service Labour Relations Board. That Board is given broad powers-broader than those typically vested in a labour board - to supervise and administer the novel system of collective bargaining created by the Public Service Labour Relations Act. The Act calls for a delicate balance between the need to maintain public services, and the need to maintain collective bargaining. Considerable sensitivity and unique expertise on the part of Board members is all the more required if the twin purposes of the legislation are to be met ».

10 Telle était d'ailleurs la situation dans l'affaire même. La Cour examine le caractère raisonable de chaque interprétation contenue dans les pièces du procès. Elle procède de cette manière non pour démontrer qu'une interprétation est plus correcte que l'autre, mais pour montrer que l'interprétation de l'agence est au moins aussi raisonnable que l'interprétation alternative suggérée par la Cour d'appel. Cf. Canadian Union of Public Employees (CUPE), Local 963 v. New Brunswick (Liquor Corp.), [1979] 2 SCR 227 at 242.
} 
droit de se tromper11». En ayant un tel "droit de se tromper », la seule situation qui permettrait les juges d'annuler la décision serait dans le cas d'une mesure « manifestement déraisonnable » (patent unreasonableness). Il s'est créé ainsi un nouveau modèle de contrôle, bien moins interventionniste que le modèle de la correction (correctness), qui jusqu'alors était le seul existant.

Le contexte historique de la décision et son argumentation ont permis de clarifier que l'objectif de la Cour suprême était celui de faire passer un message clair afin de réduire l'interventionnisme juridictionnel12. Dans ce sens, le vote du juge Dickson, qui a emporté l'adhésion de la majorité des membres du jugement, fait mention de la nécessité de respecter le choix du législateur et de laisser aux seuls soins des agences spécialisées la charge d'adopter certaines décisions. Ensuite, il fait référence à la « considérable sensibilité et expertise unique » des membres de la direction des agences, outre l'importance d'une prise de décisions rapide ${ }^{13}$. Selon les mots d'Audrey Macklin, « CUPE a transformé la base conceptuelle du contrôle substantif par le biais d'une reformulation de la relation institutionnelle entre les juges et l'état administratif (...) [la décision] signale une rupture radicale par rapport aux modes de contrôle juridictionnel existants, en plaidant en faveur d'un recul de l'interventionnisme du passé 14 ».

11 Canadian Union of Public Employees (CUPE), Local 963 v. New Brunswick (Liquor Corp.), [1979] 2 SCR 227 at 236: "The interpretation of s. 102(3) would seem to lie logically at the heart of the specialized jurisdiction confided to the Board. In that case, not only would the Board not be required to be " correct » in its interpretation, but one would think that the Board was entitled to err and any such error would be protected from review by the privative clause in s. 101 ».

12 CUPE a été décidé à la même année que Nicholson v. Haldimand-Norfolk Reg. Police Commrs., [1979] 1 S.C.R. 311, qui instaurait une ère de contrôle procédural plus intense. Mais au lieu de les considérer comme des messages contradictoires, il est possible de les concilier autour d'une interprétation selon laquelle la Cour suprême commandait un moindre contrôle substantiel et un plus grand contrôle procédural. Dans ce sens, v. DYZENHAUS, David. The politics of deference: judicial review and democracy. In: TAGGART, Michael (ed.). The Province of Administrative Law. Oxford: Hart Publishing, 1997. p. 287

13 Canadian Union of Public Employees (CUPE), Local 963 v. New Brunswick (Liquor Corp.), [1979] 2 SCR 227 at 235-236.

14 A MACKLIN, Audrey. Standard of review: the pragmatic and functional test. In: FLOOD, Coleen; SOSSIN, Lorne (Eds.). Administrative Law in Context. Toronto, Emond Montgomery, 2008. p. 206. L'autuer va au-delà: « Most importantly, [CUPE] conveyed a spirity of curial deference, a recognition that administrative decision-makers are not merely 'inferior tribunals', but specialized bodies that possess a legislative mandate to apply their expertise and experience to matters that they may be better suited to address than the 'ordinary court' » (idem, p. 204). Pour une vision contraire, en soulignant ce qui serait une renaissance de l'interventionnisme à partir des années 1980, v. LANGILLE, Brian. Judicial review, judicial revisionism and judicial responsibility. Revue Générale de Droit, Ottawa, v. 17, n. 1-2, p. 169-192, 1986. .Dans une perspective plus générique, la consécration d'une orientation déférentielle au Canada, implique une victoire de l'État Administratif. Elle constitue une valorisation des autorités administratives décentralisées, qui ont proliféré rapidement surtout à partir des années 40 du dernier siècle. La décision de la Cour suprême sous l'affaire CUPE est, ainsi, informée par une vision spécifique anti-individualiste et transmet l'intention de faciliter l'intervention étatique dans le domaine social et de promouvoir l'efficacité administrative. Dans ce sens, v. DYZENHAUS, David. The logic of the rule of law: lessons from Willis. The University of Toronto Law Journal, Toronto, v. 55, n. 3, p. 691-714, jun./sept. 2005. p. 694; DYZENHAUS, David; FOX-DECENT, Evan. Rethinking the process/substance distinction: Baker versus Canada. The University of Toronto Law Journal, Toronto, v. 51, n. 3, p. 193-242, jun./sept. 2001. p. 203: « The same 


\subsubsection{Les illustrations moins claires des droits américain et italien}

Aux États Unis et en Italie, on trouve des exemples de I'hypothèse la plus intuitive, selon laquelle la création du modèle déférent de contrôle traduit un mouvement de rétraction juridictionnelle. Cependant, l'illustration fournie par ces deux autres systèmes juridiques n'est pas si évidente. Cela se doit au fait que, dans ces cas, le contexte qui a précédé ces initiatives n'était pas d'interventionnisme clair et polémique, comme c'était le cas au Canada. En tout cas, la lecture de leurs décisions juridictionnelles les plus importantes rend évidents les appels à l'auto restriction.

\subsubsection{Le droit américain et la doctrine Chevron}

Dans le cas américain, la référence nécessaire est toujours la doctrine Chevron. Elle constitue un exemple d'initiative tendant à instituer un standard jurisprudentiel de respect aux options de l'administration publique - et, donc, un standard de l'auto restriction juridictionnelle. Au contraire du cas canadien, cependant, le contexte qui précédait Chevron n'était pas exactement celui d'une grande intervention juridictionnelle, mais d'inconsistance jurisprudentielle ${ }^{15}$. Les juges appliquaient de manière désordonnée un contrôle tantôt intense, tantôt restreint des interprétations législatives de l'administration publique, sans consacrer aucun critère précis ${ }^{16}$. Ainsi, Chevron ne vient pas mettre fin à une ère de contrôle intense de l'administration publique, à l'instar de CUPE. Son objectif est de mettre fin aux incertitudes relatives à l'intensité précise du contrôle juridictionnel qui devait avoir lieu dans ces hypothèses.

Sous cette réserve, il est clair que la célèbre décision de la Cour suprême américaine a un penchant pour l'auto restriction juridictionnelle, en transmettant une détermination aux juges américains afin qu'ils évitent les excès au moment d'effectuer leur

basic urge that led to CUPE - the urge on the part of progressive judges to install within the Court's jurisprudence a recognition of the legitimacy of the administrative state ».

15 II convient de noter toutefois l'opinion de K. WERHAN. Cet auteur interprète Chevron comme étant l'une des évidences les plus significatives du mouvement néoclassique du droit administratif américain - et considère que ce mouvement constitue une réaction aux excès de l'activisme juridictionnel des années 1960 et 70: «I argue that the Chevron decision's primary importance lies in its leading role in the Supreme Court's recent rejection of the traditional model of administrative law and its ongoing experiment to fashion a replacement paradigm. (...) I label this new paradigm the « neoclassical model », in order to suggest its apparent appeal to the Court. The neoclassical model seeks to unite the classical distinction between law and policy with a postmodern skepticism about the competence and integrity of courts to oversee agency decisionmaking. [...] The model surfaced in the late 1970s and gained momentum during the 1980s. This followed a period of intense judicial activism, roughly from the mid-1960s to mid-1970s, when courts assumed a major responsibility for ensuring the openness of agency decisionmaking processes and the soundness of the decisionmaking itself. The neoclassical model represents not only a reaction to the excesses of that activism, but also a severe overreaction that aims to overturn and replace the traditional model of administrative law as well » (in WERHAN, Keith. The neoclassical revival in administrative law. Administrative Law Review, Washington, v. 44, n. 3, p. 567-628, dec.1992. p. 567-568.

16 Sur le sujet, v. T MERRILL, Thomas W. The story of Chevron: the making of an accidental landmark. In: STRAUSS, Peter (Ed.). Administrative Law Stories. New York: Thomsom/West, 2006. p. 413. 
contrôle. C'est la raison pour laquelle, dans son extrait le plus classique, il est affirmé que dans les hypothèses dans lesquelles une question donnée n'aura pas été déjà clairement résolue par la législation pertinente (en raison d'une ambigüité ou du silence législatif), « le juge ne doit pas simplement imposer sa propre interprétation de la loi »17, mais seulement évaluer si l'interprétation fournie par l'autorité administrative est permissible. Le choix de mots laisse évidente l'exhortation à la retenue juridictionnelle ${ }^{18}$.

Ce penchant est clair aussi dans les différents passages où la Cour suprême considère les avantages institutionnels des autorités administratives dans la résolution de certaines questions, en fonction principalement de leur expertise et leur légitimité démocratique plus grande ${ }^{19}$. À la rigueur, une des contributions les plus importantes de Chevron a été de redéfinir le lien entre les juges et les autorités administratives, et d'éclaircir la distribution de compétences ${ }^{20}$. Elle a éclairci ainsi que, dans le cas des législations administrées par les agences, le rôle des juges est limité. Dans les cas d'ambigüité législative, il n'est pas nécessaire que l'interprétation administrative soit la « seule possible ou même la meilleure », dès lors qu'il lui suffit d'être permissible pour que le juge soit empêché d'y interférer ${ }^{21}$.

17 Chevron U.S.A. Inc. v. Natural Resources Defense Council, Inc., 467 U.S. 837, 843-843 (1984).

18 Dans ce sens, T. MERRILL affirme que « in the pre-Chevron period, deference to executive interpretations required special justification ; independent judgment was the default rule. Under Chevron, the court must initially establish whether the issue is suitable for independent judicial resolution; if it is not, the court automatically shifts into a deferential mode. As a result, independent judgment now requires special justification, and deference is the default rule ». MERRILL, Thomas W. Judicial deference to Executive precedent. The Yale Law Journal, New Haven, v. 101, n. 5, p. 969-1041, mar. 1992. p. 977.

19 Voir, par exemple, Chevron U.S.A. Inc. v. Natural Resources Defense Council, Inc., 467 U.S. 837, $864-866$ (1984): "Judges are not experts in the field, and are not part of either political branch of the Government. Courts must, in some cases, reconcile competing political interests, but not on the basis of the judges' personal policy preferences. In contrast, an agency to which Congress has delegated policymaking responsibilities may, within the limits of that delegation, properly rely upon the incumbent administration's views of wise policy to inform its judgments. While agencies are not directly accountable to the people, the Chief Executive is, and it is entirely appropriate for this political branch of the Government to make such policy choices - resolving the competing interests which Congress itself either inadvertently did not resolve, or intentionally left to be resolved by the agency charged with the administration of the statute in light of everyday realities ».

20 SUNSTEIN, Cass R. Law and administration after Chevron. Columbia Law Review, New York, v. 90, n. 8, p. 2071-2120, dec. 1990. p. 2078-2079: «Before the rise of the regulatory state, the allocation of authority between the executive branch and the judiciary was relatively clear: it was for the courts, not the executive, to "say what the law is ", at least in litigated cases. This understanding extended to interpretation of statutes no less than to interpretation of the Constitution. Most statutory disputes did not involve the executive branch as a party. Since government almost always acted without the mediation of a policy-making, fact-finding administrative agency, it was only natural that questions of statutory meaning would be decided by the courts. (...) All this was changed by the creation in the twentieth century of a massive administrative apparatus, which was of course a self-conscious repudiation of regulation through the judiciary. For the twentieth century reformers, courts lacked the flexibility, powers of coordination, initiative, democratic accountability, and expertise necessary to deal with complex social problems ».

21 Chevron U.S.A. Inc. v. Natural Resources Defense Council, Inc., 467 U.S. 837, 843 n.11 (1984): « The court need not conclude that the agency construction was the only one it permissibly could have adopted to uphold the construction, or even the reading the court would have reached if the question initially had arisen in a judicial proceeding ». 


\subsubsection{Le « contrôle faible » des évaluations techniques complexes en Italie}

En Italie, la meilleure référence est certainement celle du contrôle dit faible (sindacato debole). La création de ce modèle de contrôle peut être bien identifiée dans le temps, contrairement à ce qui se passe dans les cas du modèle de contrôle déférent encore aujourd'hui applicable à la discrétionnalité administrative, et du modèle de contrôle déférent qui s'appliquait auparavant aux cas de la discrétionnalité dite technique. Effectivement, le premier registre de référence du Consiglio di Stato au modèle de contrôle « faible » a eu lieu en 2001. Lors de jugements ultérieurs, le Consiglio di Stato a éclairci qu'il fallait l'appliquer aux hypothèses $d$ ' « évaluations techniques complexes » (valutazioni tecniche complessse) - concept nouveau également. Cette détermination plus précise dans le temps permet une analyse plus riche et exacte des circonstances qui ont motivé la création du modèle déférentiel.

Cela dit, il convient de reconnaître que la situation jurisprudentielle confuse que I'Italie a connue entre 1999 et 2001 rend difficile d'affirmer quel type de contrôle était appliqué auxdites évaluations techniques complexes avant les décisions rendus sous l'affaire Fornambiente22, qui a créé le modèle de contrôle faible, et sous l'affaire $R C$ Auto23, qui l'a développé. La première raison provient du fait que cette catégorie (évaluations techniques complexes) n'existait même pas dans le vocabulaire juridictionnel administratif. Elle sert à désigner les cas dans lesquels la décision administrative comprend, conjointement, des éléments de discrétionnalité pure (dans le sens classique de la mise en balance d'intérêts publics conflictuels) et ce qui est appelé discrétionnalité technique. Le problème réside sur le fait que la discrétionnalité pure était (et l'est toujours) soumise à un contrôle juridictionnel limité, tandis que la surnommée discrétionnalité technique paraissait se trouver, depuis le revirement de 1999, soumise à un contrôle illimité. Dans ce sens, à l'instar du cas américain, la décision du Consiglio di Stato sous l'affaire Formambiente ne vise pas à réformer la jurisprudence en vigueur, mais à l'éclairci. Malgré cela, comme aux États-Unis, cet éclaircissement suffit à démontrer un penchant en faveur de l'exhortation à la retenue juridictionnelle.

La première mention du contrôle faible a eu lieu sous l'affaire Formambiente24. Il s'agissait de la contestation juridictionnelle d'une décision du Comité technique forestier, qui avait empêché l'édification sur des zones soumises à des restrictions pour des raisons hydrogéologiques. La décision a été annulée par le Tribunal Administratif Régional, qui a suivi l'avis de l'expert de la justice pour conclure que, contrairement à ce que l'autorité administrative supposait, la zone en cause était propre à l'édification. Le Consiglio di Stato a annulé la décision du Tribunal Administratif Régional et a réta-

22 Cons. St., Sez. IV, 06 ottobre 2001, n. 5287.
23 Cons. St., Sez. VI, 23 aprile 2002, n. 2199.
24 Cons. St., Sez. IV, 06 ottobre 2001, n. 5287. 
bli la décision administrative. Il a affirmé que la décision comprenait une évaluation technique complexe et que, dans ces cas, le contrôle juridictionnel est «faible ", en se limitant à évaluer le caractère raisonnable des choix effectués par l'administration ${ }^{25}$. L'argumentation du Consiglio di Stato inclut une réprimande au Tribunal Administratif Régional pour être allé au-delà de ce qu'il aurait dû faire. Tel est sans doute le premier aspect pertinent vis-à-vis des buts de la présente section.

Quant au second aspect pertinent, il convient d'attirer l'attention sur le fait que le Consiglio di Stato a justifié la nécessité de l'application d'un contrôle déférent (qu'il a dénommé de "faible ») en fonction des avantages institutionnels comparatifs de l'administration publique, par rapport aux juges. Le Consiglio di Stato observe alors que la difficulté à séparer conceptuellement les considérations d'opportunité et les discussions techniques subjectivess'accentue dans les cas pour lesquels les évaluations présentent une nature d'objective complexité, liées à l'importance des intérêts en jeu. Dans ces cas, le législateur attribue normalement du pouvoir décisoire à des entités de l'administration « dotées d'une particulière légitimation, à la lumière de leur composition et de leur qualification technique 26 ». II convient de noter que le Consiglio di Stato parle de " particulière légitimation », afin de souligner également une plus grande légitimation de l'Administration que celle du juge, en ce qui concerne la prise de ces décisions importantes. Tel était aussi le cas du Comité technique forestier.

25 Cons. St., Sez. IV, 06 ottobre 2001, n. 5287, point 8: « Il giudice è stato qui chiamato a pronunciarsi sull'impugnazione di una delibera di autorizzazione che era stata presa sulla base di una valutazione di natura complessa, che ha abbracciato la consistenza del vincolo idrogeologico gravante sull'area, l'estensione e la specifica collocazione del terreno della società e le possibili conseguenze che sarebbero derivate dal disboscamento a scopo edificatorio: in breve, sia considerazioni collegate ad un giudizio tecnico opinabile sia considerazioni di opportunità amministrativa. Nel dirimere una controversia annoverabile, secondo tradizione, in quelle collegate al controllo della c.d. discrezionalità tecnica, il giudice non si è limitato a censurare le valutazioni tecniche (non giuridiche) che erano apparse sicuramente inattendibili ed irragionevoli, ma ha direttamente sostituito la valutazione tecnica sviluppata nel processo a quella effettuata dall'amministrazione. Si è compiuto, a ben vedere, un controllo di tipo «forte », che si traduce in un potere sostitutivo del giudice, il quale si spinge fino a sovrapporre la propria valutazione tecnica opinabile a quella dell'amministrazione. Mentre il controllo di tipo « debole » avrebbe richiesto che le cognizioni tecniche acquisite grazie al consulente fossero utilizzate solo allo scopo di effettuare un controllo di ragionevolezza e coerenza tecnica della decisione amministrativa. Ebbene, nel caso di specie, un controllo di tipo « forte » non poteva legittimamente svolgersi ».

26 Cons. St., Sez. IV, 06 ottobre 2001, n. 5287, point 9-10: «In secondo luogo, deve tenersi in debito conto che la difficoltà di separare concettualmente tra opinabilità ed opportunità amministrativa è accentuata proprio nei casi in cui la valutazione presenta caratteri di obiettiva complessità, collegata alla rilevanza di interessi di rango primario ; interessi protetti da norme costituzionali destinate a prevalere nel giudizio di bilanciamento che le contrappone all'interesse dedotto dal ricorrente, anch'esso di sicura ascendenza costituzionale grazie al disposto dell'art.113 Cost.. L'emersione di siffatti interessi, inoltre, induce il legislatore primario ad affidare il potere di provvedere ad articolazioni dell'amministrazione che si assumono dotate di una peculiare legittimazione, alla luce della composizione e qualificazione tecnica. (...) E', infine, univoca la scelta legislativa che, in coerenza col principio di buon andamento dell'attività amministrativa, demanda la valutazione ad un organo che si configura come dotato di una specifica competenza, funzionale alla corretta salvaguardia del vincolo. In questo settore la legge ha assunto al rango di interesse pubblico un interesse che ha anche valenza tecnico-scientifica e ne ha attribuito la cura ad una particolare autorità amministrativa ». 
Les considérations institutionnelles qui ont justifié cette restriction juridictionnelle ont été développées dans plusieurs affaires ultérieures. Dans ces affaires, l'argumentation a toujours été dans le sens d'expliquer pourquoi un contrôle déférentserait préférable ou plus opportun ${ }^{27}$. Il devient donc évident qu'en Italie, le contrôle faible a été créé pour limiter l'intervention juridictionnelle sur certaines décisions administratives.

\subsection{L'hypothèse contre-intuitive: le modèle déférent pour l'élargis- sement de l'intervention}

La section ci-dessus a démontré que la création d'un modèle de contrôle déférent peut servir à faire passer un mouvement de rétraction juridictionnelle. La perspective du droit français en est totalement distincte. Dans ce pays, le modèle déférent de contrôle juridictionnel (appelé contrôle minimum ou restreint) a été créé pour permettre une intervention juridictionnelle réduite aux matières qui auparavant n'étaient soumises à aucun contrôle juridictionnel. Cela signifie que la fonction historique du contrôle restreint en France est celle de permettre une augmentation du contrôle juridictionnel, bien que cette augmentation n'implique pas l'adoption d'un contrôle non déférent. En d'autres mots, la création du modèle de contrôle déférent en France ne traduit pas un mouvement de rétraction, mais d'avance, d'intensification ou d'élargissement du contrôle exercé par le juge sur l'administration publique.

\subsubsection{La création du modèle de contrôle restreint en 1961 en France}

Le modèle de contrôle restreint a été adopté pour la première fois sous l'affaire Lagrange, en 196128. Sa création a permis au Conseil d'État de soumettre les décisions administratives relatives à l'équivalence de fonctions exercées par des agents publics à un certain contrôle juridictionnel. La matière relevait auparavant d'une large discrétionnalité administrative, en n'étant soumise à aucune surveillance juridictionnelle. À partir de l'affaire Lagrange, la jurisprudence administrative peut au moins censurer les erreurs les plus grossières ou les plus évidentes de l'administration publiques - appelées « erreurs manifestes d'appréciation ».

27 Voir, par exemple, Cons. St., Sez. VI, 23 aprile 2002, n. 2199, point 1.3.1, dans lequel le Consiglio di Stato affirme que l'autorité de la concurrence a une composition et une qualification technique particulières, outre le fait de bénéficier d'un haut degré d'autonomie et d'indépendance, raisons pour lesquelles le contrôle juridictionnel exercé sur ses évaluations techniques complexes devait se limiter à une évaluation de leur caractère raisonnable. Voir aussi Cons. St., Sez. VI, 01 ottobre 2002, n. 5156, points 6 et 9, dans lesquels le Consiglio di Stato reproche au TAR de Lazio d'avoir effectué un contrôle fort sur l'existence de position dominante, évaluation qui doit être réservée à l'autorité de la concurrence, autorité " neutre » et « légitime ", dotée de caractéristiques institutionnelles qui la rendent mieux adaptée que les juges pour effectuer des jugement techniques et des pronostics.

28 CE, 15 février 1961, Lagrange. R. ODENT conteste cette information dominante, en affirmant que ce contrôle avait déjà été appliqué lors de la décision CE 13 novembre 1953, Denizet (in Contentieux administratif, Paris, Institut d'Études Politiques de Paris, 1971, pp. 1560 et suivantes). 
Les deux décennies suivantes ont été marquées par des mouvements similaires dans des divers domaines de l'action administrative. Quelques exemples peuvent illustrer cette affirmation. En 1973, le Conseil d'État a décidé de soumettre à un contrôle restreint les interdictions administratives de publications étrangères ${ }^{29}$. Ces mesures étaient considérées avant comme l'expression de la haute police administrative et, par conséquent, échappaient à tout contrôle juridictionnel. Le changement de cette jurisprudence a suivi l'exhortation du commissaire du gouvernement Braibant. Dans ses conclusions, il a observé qu'à l'époque déjà, le contrôle restreint paraissait totalement disséminé, et a suggéré au Conseil d'État d'étendre cette solution à la police administrative relative aux publications étrangères, notamment en fonction du risque de violation de libertés fondamentales ${ }^{30}$. Deux années plus tard, le contrôle restreint a également commencé à atteindre les appréciations d'existence d'une menace à l'ordre public dans le cas de la présence d'un étranger, d'origine non communautaire, sur le sol français 31 - situation qui elle-aussi échappait à la surveillance juridictionnelle32. En 1978, en revenant sur une extensive jurisprudence dans le sens contraire ${ }^{33}$, le Conseil d'Etat admet pour la première fois l'analyse de l'erreur manifeste en cas de sanction disciplinaire ${ }^{34}$.

29 CE, 2 nov. 1973, SA Librairie François Maspero, Requête nº 82590.

30 Après avoir commenté le stage dans lequel se trouvait la jurisprudence en la matière (échappant jusqu'alors au contrôle juridictionnel), le commissaire du gouvernement $\mathrm{M}$. Braibant a conclu à ceci: « Le moment nous paraît venu d'aller au delà de cette jurisprudence traditionnelle. Sans doute ne peut-il être question pour le juge de substituer son appréciation à celle de l'autorité administrative lorsque cette autorité dispose d'un pouvoir discrétionnaire, que vous définissez précisément comme un pouvoir d'appréciation. Mais il y a déjà douze ans que vous avez étendu votre contrôle en la matière en utilisant la notion d'erreur manifeste d'appréciation : le pouvoir discrétionnaire comporte le droit de se tromper, mais non celui de commettre une erreur manifeste c'est-à-dire à la fois apparente et grave. Le champ d'application de cette jurisprudence nouvelle c'est [sic] peu à peu étendu aux domaines les plus variés, de sorte que l'on peut se demander si elle n'a pas aujourd'hui un caractère général. Vous l'avez appliqué à des appréciations aussi difficiles que celles de la valeur d'un fonctionnaire ou de la nocivité d'un produit. Mais vous ne l'avez pas encore employé dans le domaine des mesures de police sinon seulement vous n'avez jamais annulé une telle mesure sur ce terrain, mais vous n'en avez même pas réservé expressément la possibilité. Seul le tribunal administratif de Paris s'est jusqu'à présent engagé dans cette vois en évoquant l'éventualité d'une annulation pour une erreur manifeste d'appréciation à propos de l'interdiction faite à un étranger de pénétrer sur le territoire français (...) Nous vous proposons de faire vôtre cette solution. Le domaine de la police est sans doute celui dans lequel le contrôle du juge est le plus nécessaire parce qu'il touche a des libertés fondamentales ». V. conclusions du commissaire du gouvernement BRAIBANT sous la décision CE, 2 nov. 1973, SA Librairie François Maspero, Requête n 82590.

31 CE, 3 fév. 1975, Min. intérieur c. Pardov, Requête n 94108.

32 V., dans ce sens, par exemple, CE 13 juin 1952, Meyer.

33 L'arrêt de principe était CE 22 nov. 1967, Dlle Chevreau, décidé après la création du modèle de contrôle restreint en 1961. En l'espèce, le Conseil d'État a refusé de vérifier l'adéquation de la sanction disciplinaire maximum appliquée par l'Assistance Publique de Paris à une faute prétendument non lourde d'un infirmier. L'application d'un contrôle restreint avait été suggérée par le commissaire du gouvernement Kahn, mais n'a pas été acceptée par le Conseil d'État. L'orientation a été confirmée ultérieurement dans la décision CE 28 avril 1976, Gorin T. ; CE 2 juin 1976, Dame de Robert ; CE 1er octobre 1976, Soucasse.

34 CE Sect. 9 juin 1978, Lebon, Requête n05911. II s'agissait de l'examen de la proportionnalité de la sanction de la mise en retraite obligatoire d'un instituteur accusé de comportements contraires à la morale et aux bonnes moeurs à l'encontre d'élèves de sa classe. 
Dans ce cas, le commissaire du gouvernement Bruno Genevois a repris les raisons qui avaient été écartées dans les affaires précédentes par le Conseil d'État, afin d'élargir son contrôle, en suggérant que « le refus d'exercer tout contrôle n'était pas en harmonie avec l'évolution général du [contrôle du Conseil d'État] sur l'exercice d'un pouvoir discrétionnaire par l'administration 35 ».

Dans ces premières affaires, il est possible de voir clairement le penchant historique du modèle de contrôle restreint : une forme de rendre viable l'élargissement de la surveillance juridictionnelle. Dans les cas canadien, américain et italien cités ci-dessus, l'adoption d'un modèle de contrôle déférent a exigé de l'effort et de l'énergie des juges afin de justifier pourquoi un contrôle plus fort, non déférent n'était pas adopté. Toute l'argumentation des juges a été bâtie dans ce sens. Au contraire, en droit français, le modèle de contrôle restreint a été créé pour permettre une plus grande intervention de juge dans les cas qui échappaient auparavant au contrôle juridictionnel. Pour cette raison, l'effort argumentatif des commissaires du gouvernement était dirigé entièrement vers la fin de convaincre qu'il fallait opérer davantage de contrôle, dès lors que cela était opportun et juridiquement possible. En d'autres mots, au Canada, aux États-Unis et en Italie, il a été nécessaire d'expliquer pourquoi il fallait adopter un contrôle déférent, $a u$ lieu d'un contrôle non déférent; en France, il a été nécessaire d'expliquer pourquoi adopter un contrôle déférent, au lieu d'absence de contrôle. Dans ce contexte, un modèle de contrôle dans lequel l'intervention des juges est limitée sert comme instrument pour faciliter l'introduction de la surveillance juridictionnelle, en la rendant plus légère, moins abrupte - et, donc, plus facilement acceptable. La stratégie s'est révélée couronnée de succès. La surveillance juridictionnelle (par le biais du contrôle normal ou restreint) est aujourd'hui totalement disséminée en droit français. Les cas d'insubordination de décisions administratives au contrôle juridictionnel se trouvent réduits à des hypothèses très rares et quasi anecdotiques 36 .

\footnotetext{
35 En affirmant que le modèle de contrôle de l'erreur manifeste se trouvait à stade très généralisé, le commissaire a conclu que le Conseil d'État devait commencer à l'adopter: « Le refus d'exercer tout contrôle n'est pas en harmonie avec l'évolution générale de votre contrôle sur l'exercice par l'administration d'un pouvoir discrétionnaire. (...) À l'époque ou vous avez rendu la décision Demoiselle Chevreau, la notion d'erreur manifeste était encore peu développée. (...) Mais, depuis 1967, la théorie de l'erreur manifeste a été généralisée et s'applique dans les domaines les plus divers (...) On s'explique dès lors assez mal le maintien de votre jurisprudence traditionnelle » (Conclusions du commissaire du gouvernement Bruno Genevols sous la décision CE Sect. 9 juin 1978, Lebon, Requête no 05911).

36 Le " contrôle infra-minimum » s'applique aujourd'hui à des décisions telles que la concession d'honneurs (CE 27 nov. 2000, Ass. comité tous frères ; CE, 10 déc. 1986, Loredon: AJDA 1987, p.91.) ; le mode de gestion de services publics (CE, 27 nov. 2002, Sté d'intérêt collectif agricole d'électricité (SICAE) de la région de Péronne: AJDA 2003, p. 575 ; CE, 10 jan. 1992, Assoc. des usagers de l'eau de Peyreleau, RFDA 1992, p.346) et les décisions des jurys de concours publics (CE 16 mai 2001, de Nale ; CE 03 nov. 1982, Goure ; CE 15 avril 1983, Bannay ; CE 20 mai 1994, Mlle Semetey; CE 22 févr. 1995, Girardot).
} 


\section{L'ÉVOLUTION HISTORIQUE DU MODĖLE DE CONTRÔLE DÉFÉ- RENT}

La section précédente a examiné les circonstances historiques et les arguments des juges dans des jugements-clé afin d'identifier le penchant historique des modèles déférents de contrôle dans chacun des systèmes juridiques étudiés. Dans ce second pas, l'idée est d'examiner l'évolution connue par chacun de ces modèles au cours des décennies ultérieures à leur création.

Les droits canadien et américain offrent des illustrations du succès du modèle déférentiel (2.1). Au Canada, le modèle du caractère raisonnable a connu une ascension progressive, au point de devenir aujourd'hui le modèle de contrôle les plus commun. Un peu moins clairement, aux États-Unis, l'orientation déférente de Chevron subsiste comme règle, même devant des réformes (ou éclaircissements) apportés par la doctrine au jugement de l'affaire Mead.

Les droits italien et français, pour leur part, offrent des exemples contraires, de situations dans lesquelles les modèles de contrôle déférentiels ont perdu progressivement leur prestige au fil du temps (2.2). En France, après une phase initiale de diffusion, le modèle de contrôle déférentiel a perdu progressivement son prestige en bénéfice d'un contrôle plus intense, appelé contrôle normal, non déférent. Le cas italien est le paradigme le plus flagrant de l'échec : le contrôle dit faible a été abandonné trois ans seulement après avoir été créé.

\subsection{L'hypothèse de dissémination du modèle déférent}

Encore une fois, le Canada apporte l'exemple le plus clair d'une tendance. Ici, l'illustration concerne l'ascension du modèle de contrôle déférentiel. Inexistant jusqu'en 1979, l'orientation déférente s'est disséminée rapidement et, malgré les réformes internes, s'est établi comme dominant (2.1.1). Aux États-Unis, l'illustration du succès est moins claire, mais paraît être perceptible aussi (2.1.2).

\subsubsection{L'illustration claire du droit canadien: le déclin du modèle de la correction}

Au long de son histoire jurisprudentielle, le droit canadien a connu différents modèles déférents de contrôle. Le premier à être créé a été le modèle du caractère manifestement déraisonnable, en 1979, dans le cas CUPE37. Pendant presque 20 ans, les seuls modèles de contrôle ont été le modèle non déférent de la correction et le modèle ultra-déférent du caractère manifestement déraisonnable. En 199738, un troisième

\footnotetext{
37 Canadian Union of Public Employees (CUPE), Local 963 v. New Brunswick (Liquor Corp.), [1979] 2 SCR 227 at 235-236.

38 Canada (Director of Investigation and Research) v. Southam Inc., [1997] 1 SCR 748, para. 54-56.
} 
modèle de contrôle a été créé, celui du caractère raisonnable simple. Enfin, en 200839, la Cour suprême a réformé le système, en mélangeant les deux modèles de contrôle restreint en un seul modèle déférentiel, en l'appelant simplement modèle du caractère raisonnable.

Indépendamment d'un tel va-et-vient quant au nombre de modèles déférents, la déférence juridictionnelle en soi a fait l'objet d'une forte évolution en droit canadien 40 . Selon les mots de David Mullan, elle est aujourd'hui une « valeur transcendantale » en droit administratif canadien ${ }^{41}$. Aujourd'hui, il subsiste très peu de choses du contexte fortement interventionniste qui a motivé la révolution sous l'affaire CUPE, en 1979. Les hypothèses d'application des différents modèles déférents de contrôle juridictionnel se sont multipliées et les cas d'application du modèle de la correction ont été drastiquement réduits. Aujourd'hui, ce-dernier ne s'applique que quasi exclusivement pour le contrôle de décisions administratives qui comprennent une forte sensibilité juridiques, comme celles de nature constitutionnelle ou relatives à des droits fondamentaux ou de l'homme42.

En tant que l'une des expressions les plus claires du prestige du modèle de contrôle juridictionnel déférent, il est possible de citer la proposition du juge Binnie, de la Cour suprême canadienne, sous l'affaire Dunsmuir. De manière générale, la réforme apportée dans cette affaire visait à optimiser le système de contrôle juridictionnel au Canada, en réduisant sa complexité et en élargissant son aspect opérationnel. La réforme a fortement impacté la procédure contextuelle de détermination de l'intensité du contrôle juridictionnel - appelée avant « analyse pragmatique et fonctionnelle » et, à partir de Dunsmuir, seulement « analyse du modèle de contrôle juridictionnel ». En tant qu'élément additionnel pour la simplification du système, le juge Binnie a suggéré l'adoption de quelques présomptions. Cela éviterait d'aborder des analyses complexes dans l'ensemble des cas. Ces analyses ne seraient nécessaires que lorsque l'une des

39 Dunsmuir v. New Brunswick, [2008] 1 SCR 190, para. 45.

40 II ne semble pas correct d'interpréter l'élimination du modèle de contrôle du caractère manifestement déraisonnable comme un pas en direction d'un plus grand interventionnisme juridictionnel. La Cour suprême même a cherché à éviter ce raisonnement. V. Dunsmuir v. New Brunswick, [2008] 1 SCR 190, para. 48: "The move towards a single reasonableness standard does not pave the way for a more intrusive review by courts and does not represent a return to pre-Southam formalism ».Dans le même sens, v. aussi J CRAIG, Jared. Defending city hall after Dunsmuir. Alberta Law Review Edmonton, v. 46, n. 1, p. 275-297, nov. 2008. p. 292.

41 MULLAN, David J. Recent developments in administrative law - The apparent triumph of deference! Canadian Journal of Administrative Law and Practice, v. 12, n. 2, p. 191-, 1998. p. 192. L'auteur affirme aussi: « the Supreme Court of Canada in a substantial number of its recent prominent administrative law judgments has been prepared to accord a significant degree of autonomy to and respect for the operational imperatives of administrative tribunals. Moreover, this philosophy or approach has extended beyond typical standard of review cases to a broad range of other situations involving the relationship between the courts and the administrative process » (ibidem, p. 204).

42 V. JACOBS, Laverne. Developments in administrative law: the 2007-2008 term - The impact of Dunsmuir. Supreme Court Law Review, Ottawa, v. 43, n. 2, p. 1-34, dec. 2008. p. 17. 
parties intéressées cherchait à démontrer que la présomption ne s'appliquait pas à l'espèce ${ }^{43}$.

La présomption principale, conformément à la proposition du juge Binnie, devait être celle prévoyant que le modèle de contrôle serait en général celui du caractère raisonnable, lorsqu'il s'agirait d'une décision d'une autorité administrative spécialisée44. La partie qui plaiderait en faveur de l'application du modèle de la correction devrait alors démontrer que la décision contrôlée se revêtait d'une sensibilité juridique particulière 45 .

Bien que cette proposition n'ait pas été adoptée par la majorité de la Cour suprême, il semble légitime d'admettre que, en soi, elle offre déjà un témoignage au sujet de la grande dissémination (et même la prédominance) du modèle du caractère raisonnable. L'idée consistait justement à officialiser cette prédominance, avec l'objectif de simplifier la tâche de détermination de l'intensité du contrôle juridictionnel applicable dans un cas concret.

\subsubsection{L'illustration moins claire du droit américain: le mouvement pendulaire}

Dans le cas des États Unis, la dissémination du modèle déférentiel de contrôle juridictionnel n'est pas aussi claire. Dans le cas de la doctrine Chevron, le grand point de référence de son évolution jurisprudentielle repose sur la restriction de ses termes originels sous l'affaire Mead. Dans cette affaire, la Cour suprême affirme que le test de Chevron est applicable uniquement aux hypothèses dans lesquelles il est possible d'identifier une délégation législative afin que les autorités administratives se prononcent avec force de loi.

\footnotetext{
43 Dunsmuir v. New Brunswick, [2008] 1 SCR 190, para. 145: "The present incarnation of the "standard of review » analysis requires a threshold debate about the four factors (non-exhaustive) which critics say too often leads to unnecessary delay, uncertainty and costs as arguments rage before the court about balancing expertise against the « real » nature of the question before the administrator, or whether the existence of a privative clause trumps the larger statutory purpose, and so on. And this is all mere preparation for the argument about the actual substance of the case. While a measure of uncertainty is inherent in the subject matter and unavoidable in litigation (otherwise there wouldn't be any), we should at least (i) establish some presumptive rules and (ii) get the parties away from arguing about the tests and back to arguing about the substantive merits of their case ".

44 Dunsmuir v. New Brunswick, [2008] 1 SCR 190, para. 146: « The going-in presumption should be that the standard of review of any administrative outcome on grounds of substance is not correctness but reasonableness (" contextually » applied). The fact that the legislature designated someone other than the court as the decision maker calls for deference to (or judicial respect for) the outcome, absent a broad statutory right of appeal. Administrative decisions generally call for the exercise of discretion. Everybody recognizes in such cases that there is no single « correct » outcome ».

45 Dunsmuir v. New Brunswick, [2008] 1 SCR 190, para. 147: « An applicant urging the non-deferential « correctness » standard should be required to demonstrate that the decision under review rests on an error in the determination of a legal issue not confided (or which constitutionally could not be confided) to the administrative decision maker to decide, whether in relation to jurisdiction or the general law. Labour arbitrators, as in this case, command deference on legal matters within their enabling statute or on legal matters intimately connected thereto $»$.
} 
L'avènement de Mead n'a pas besoin nécessairement d'être interprété comme une rétraction de la doctrine Chevron, mais plutôt comme un éclaircissement de ses réelles hypothèses d'application. Dans ce sens, la doctrine Chevron reste en vigueur presque 30 ans après son élaboration. En outre, même dans les cas dans lesquels elle n'a pas à s'appliquer, une autre doctrine issue de l'affaire Skidmore peut s'appliquer, laquelle détermine que soit donné du poids aux décisions administratives dans la mesure de leur pouvoir de persuasion. Cela signifie une expansion de l'orientation déférentielle (selon des gradations variées) pour presque toutes les interprétations législatives réalisées par les autorités administratives.

En dehors du champ étroit des interprétations législatives, l'orientation déférentielle est dominante aussi. L'Administrative Procedure Act (APA) réserve à des hypothèses très exceptionnelles le modèle de contrôle non déférentiel (dit de novo). Bien plus nombreuses, en revanche, ces sont les hypothèses d'application des modèles d'évidences substantielles et du caractère arbitraire - tous les deux déférents, à un point tel de rendre commun la demande doctrinale pour qu'ils soient fusionnés avec le second pas du test de Chevron.

II faut en conclure que, bien que n'étant pas aussi claire que le cas canadien, dans l'expérience des États-Unis il paraît exister aussi une dissémination et la prédominance des modèles déférents de contrôle juridictionnel 46.

46 Dans une perspective plus générique, la doctrine américaine souligne le caractère pendulaire de la gradation de l'intervention juridictionnelle dans les décisions de l'administration publique. En tournant le regard vers les 100 dernières années du droit administratif de ce pays, il est possible de noter que les mouvements de rétraction juridictionnelle se succèdent à des périodes d'un plus grand activisme. Pour un approfondissement historique, v. WERHAN, Keith. The neoclassical revival in administrative law. Administrative Law Review, Washington, v. 44, n. 3, p. 567-628, dec.1992. Dans un résumé rapide, la première mention à faire est celle qu'il appelle « early modern approach ». En vigueur dans les premières décennies du XXème siècle, il se caractérise par le formalisme juridictionnel et par le penchant contraire à l'administration publique - d'où la tendance vers l'accentuation du contrôle juridictionnel. Ce premier moment est dépassé par la suite en fonction de la consolidation du New Deal, programme qui multiplie la quantité d'autorités administratives décentralisées, leur importance et leur prestige. En se fondant sur l'institutionnalisme réaliste, a lieu alors le mouvement que Werhan appelle de "Legal Process ». Cette période se caractérise par une forte rétraction juridictionnelle, fondées sur une plus grande expertise et sur l'adaptation institutionnelle des autorités administratives. À partir des années 1960 et pendant environ vingt ans, se produit une nouvelle oscillation du pendule, donnant lieu au mouvement du «Interest Representation ». Caractérisé par une forte mécroyance dans un gouvernement d'experts et poussé par la grande préocupation avec le phénomène de la capture des autorités administratives par les industries régulées, le mouvement donne lieu à une nouvelle période d'activisme juridictionnel. Cette orientation est particulièrement visible dans le contrôle procédural, dans lequel il est fait allusion à une « hard look doctrine ». V. GARRY, Patrick M. Judicial review and the 'hard-look' doctrine. The Nevada Law Journal, Las Vegas, v. 7, n. 1, p. 151-170, sept./dec. 2006; C. SUNSTEIN, Cass R. Deregulation and the hard-look doctrine. The Supreme Court Review, Chicago, v. 24, p. 177-213, 1983. A partir de la fin des années 1970 - et principalement dans les années 1980 - il y a la période néoclassique, qui reprend la distinction entre law et policy qui était chère au modèle classique et rétablit la tendance déférentielle. Des affaires comme Chevron et Heckler v. Chaneyet les restrictions réalisées dans les règles relatives à l'intérêt à agir sont quelques unes de leurs expressions majeures. Sur la renaissance du néoclassissisme, voir aussi SUNSTEIN, Cass R. Law and administration after Chevron. Columbia Law Review, New York, v. 90, n. 8, p. 2071-2120, dec. 1990. À la suite de ce résumé de la nature pendulaire de la rétraction juridictionnelle aux États Unis, il n'est pas totalement claire qu'il soit possible de souligner le succès de l'orientation déférente. Cependant, cette orientation est clairement dominante 


\subsection{L'hypothèse de déclin ou d'abandon du modèle déférent}

Dans d'autres systèmes juridiques, le modèle déférent de contrôle n'a pas mérité un pareil destin. En Italie, le contrôle dit faible n'a duré que trois ans, en étant très vite abandonné par le Consiglio di Stato (2.2.1). En France, le contrôle restreint cède progressivement de la place au contrôle normal, même s'il est encore utilisé dans certaines hypothèses (2.2.2).

\subsubsection{L'abandon total du contrôle faible en droit italien}

Créé en 2001 sous l'influence du droit américain, le modèle de contrôle faible a eu une existence très éphémère en droit italien. Depuis sa conception, il a fait l'objet de fortes critiques de la doctrine, qui y voyait une mise en cause de la protection juridictionnelle. En 2004, avant de compléter ses courts 3 ans, le modèle de contrôle faible a été abandonné par le Consiglio di Stato.À partir de la décision du Consiglio di Stato sous l'affaire Buoni Pasto47, les évaluations techniques complexes ont commencé à être soumises non plus à un contrôle « faible », mais à un contrôle «plein et particulièrement pénétrant ». Initialement, la plus haute juridiction administrative italienne a voulu faire croire qu'il s'agissait d'une simple modification nominative : ses décisions précédentes ayant été mal interprétées, le contrôle faible signifierait simplement un contrôle non substitutif. En raison justement d'un tel « malentendu », il serait opportun de ne plus faire référence aux dénominations « fort » et « faible 48 ».

aujourd'hui. Pour une image pendulaire dans I'histoire jurisprudentiel américain du XIXème siècle également, v. WOOLHANDLER, Ann. Judicial deference to administrative action - A revisionist history. Administrative Law Review, Washington, v. 43, p. 197-223, 1991. Sur le thème du penchant relatif au contrôle juridictionnel, v. aussi L. JAFFE, Louis L. The right to judicial review I. Harvard Law Review, New Haven, v. 71, n. 3, p. 401-437.

47 Cons. St., Sez. VI, 02 marzo 2004, n. 926, Buoni Pasto. Il s'agissait de la condamnation imposée par l'autorité de la concurrence italienne à différentes entreprises du marché du coupon-déjeuner, accusées de former un cartel dans l'appel d'offres réalisé par la Consip (entreprise publique) pour l'achat de tickets pour les agents de l'administration publique. Les entreprises auraient réalisé des ententes afin de fixer le niveau de réduction et la répartition des objets de l'appel d'offres.

48 Cons. St., Sez. VI, 02 marzo 2004, n. 926, 3.3: «Tale impostazione esclude ogni vulnus alla tutela giurisdizionale e le stesse censure proposte dalle imprese sembrano incentrarsi maggiormente sulla (criticata) limitazione del controllo giurisdizionale ad un sindacato « di tipo debole », emergente dai principi in precedenza affermati dalla Sezione, senza la verifica in concreto del controllo svolto dal giudice amministrativo. In realtà, con l'espressione sindacato « di tipo debole » la Sezione non ha inteso limitare il proprio potere di piena cognizione sui fatti oggetto di indagine e sul processo valutativo, mediante il quale l'Autorità applica alla fattispecie concreta la regola individuata. Con tale espressione si è inteso porre solo un limite finale alla statuizione del giudice, il quale, dopo aver accertato in modo pieno i fatti ed aver verificato il processo valutativo svolto dall'Autorità in base a regole tecniche, anch'esse sindacate, se ritiene le valutazioni dell'Autorità corrette, ragionevoli, proporzionate ed attendibili, non deve spingersi oltre fino ad esprimere proprie autonome scelte, perché altrimenti assumerebbe egli la titolarità del potere. Il giudice non può sostituirsi ad un potere già esercitato, ma deve solo stabilire se la valutazione complessa operata nell'esercizio del potere debba essere ritenuta corretta sia sotto il profilo delle regole tecniche applicate, sia nella fase di « contestualizzazione » della norma posta a tutela della concorrenza che nella fase di raffronto tra i fatti accertati ed il parametro « contestualizzato ». 
Des décisions ultérieures ont précisé que la rupture était beaucoup plus grave. Tandis qu'auparavant la déférence était justifiée au moyen d'arguments institutionnels (comme la neutralité, l'autonomie et l'indépendance des autorités de régulation), à partir de 2004 les mêmes arguments servent à justifier un contrôle non déférent ${ }^{49}$. Tandis qu'auparavant il était fait mention d'un contrôle limité en fonction de l'interprétation de " concepts juridiques indéterminés », à partir de 2004 il est fait affirmation explicite d'un contrôle plein aussi à leur égard50. Tandis qu'auparavant il était fait mention d'un contrôle sur le caractère raisonnable, à partir de 2004 il est affirmé que les juges peuvent « réévaluer les choix techniques réalisés ».En résumé, l'orientation déférente antérieure est dépassée. Le contrôle restreint redevient applicable uniquement aux décisions administratives prises dans l'exercice d'une discrétionnalité administrative pure (non technique).

\subsubsection{Le modèle de contrôle restreint français comme "rituel de passage »}

En droit français, le déclin du modèle de contrôle juridictionnel déférent (appelé restreint ou minimum) est plus subtil, mais non moins intéressant. Au cours des premières décennies de son existence (après la création, sous l'affaire Lagrange, en 1961), le modèle de contrôle restreint a connu une grande expansion, en réduisant les cas d'absence de contrôle juridictionnel (appelé contrôle infra-minimum) à quelques rares hypothèses. Toutefois, par la suite, le contrôle restreint a cédé peu à peu de la place au contrôle normal. Plusieurs domaines d'action administrative ont fait l'objet d'une intensification du contrôle juridictionnel, en passant sous le régime du contrôle normal. Cela a conduit plusieurs auteurs à soutenir la nature " transitoire » du modèle de contrôle restreint, constituant un premier pas en direction de l'adoption ultérieur d'un contrôle non déférent.

Déjà dans les années 70, ce caractère transitoire du modèle de contrôle restreint s'est fait remarquer. En 1976, le Conseil d'État a transféré vers le modèle de contrôle

49 Cons. St., Sez. VI, 02 marzo 2004, n. 926, 3.3: « Il fatto che si tratti di un'Autorità posta al di fuori del circuito dell'indirizzo politico rende ancor più necessario che il sindacato del giudice sia pieno, ma non anche che al giudice sia consentito di sostituirsi all'Autorità nell'esercizio del potere ad essa spettante ».

50 V. Cons. St., Sez. VI, 02 marzo 2004, n. 926, 3.3 ; Cons. St., Sez. VI, 03 febbraio 2005, n. 280, point 2.1 ; Cons. St., Sez. VI, 08 febbraio 2007, n. 515, 4. Dans cette dernière décision, il est possible de lire: « Infatti, con riferimento alle valutazioni tecniche, anche quando riferite ai c.d. " concetti giuridici indeterminati », la tutela giurisdizionale, per essere effettiva, non può limitarsi ad un sindacato meramente estrinseco, ma deve consentire al giudice un controllo intrinseco, avvalendosi eventualmente anche di regole e conoscenze tecniche appartenenti alla medesima scienza specialistica applicata dall'amministrazione (Cons. St., VI, n. 2199/2002 Rc Auto ; n. 5156/2002 Enel/Infostrada). II sindacato del giudice amministrativo. è quindi pieno e particolarmente penetrante e si estende sino al controllo dell'analisi (economica o di altro tipo) compiuta dall'Autorità, potendo sia rivalutare le scelte tecniche compiute da questa, sia applicare la corretta interpretazione dei concetti giuridici indeterminati alla fattispecie concreta in esame (Cons. St., VI, n. 926/2004, Buoni pasto Consip) ». Au contraire de ce que les mentions des décisions de 2002 mènent à croire, elles n'appuient pas les affirmations qui y sont exposées. 
normal le contentieux relatif aux décisions du ministre ou des inspecteurs du travail d'autoriser le licenciement des salariés qui jouissent d'une stabilité en raison de la fonction représentative qu'ils exercent. Le sujet avait été soumis au contrôle restreint huit années auparavant seulement ${ }^{51}$. D'autres cas classiques ont tardé un peu plus. Le contentieux relatif à l'interdiction de publications étrangères, qui avait été déplacé vers le champ du contrôle juridictionnel restreint sous l'affaire Maspéro, en 197352, alors qu'auparavant il ne faisait l'objet d'aucun contrôle, a été soumis au modèle de contrôle normal sous l'affaire Association Ekin, en 199753. II s'agissait d'une évolution surprenante dans une matière considérée auparavant comme relevant de la haute policeadministrative et, par conséquent, réfractaire à l'intervention juridictionnelle. Un dernier et curieux exemple de la transition du modèle de contrôle restreint vers celui du contrôle normal, est celui de l'équivalence de fonctions publiques - objet de l'affaire Lagrange, dans laquelle le modèle de contrôle restreint a été créé. Depuis une décision du Conseil d'État en 1994, cette matière est soumise au modèle de contrôle juridictionnel non déférent, dit normal54.

Afin de compléter le tableau, il convient d'ajouter une circonstance surprenante: dans aucun cas del'histoire jurisprudentielle française, le contrôle restreint n'a été adopté dans des domaines qui originellement étaient soumises à un contrôle normal, non déférent. C'est-à-dire : I'adoption d'un contrôle déférent n'a jamais signifié, dans l'histoire jurisprudentielle française, un mouvement de rétraction juridictionnelle. Il s'agissait toujours et invariablement d'une action d'intensification du contrôle juridictionnel réalisé antérieurement. Cela démontre que l'évolution historique du contrôle juridictionnel en France est clairement unidirectionnelle, en allant toujours dans le sens de l'élargissement de la surveillance des juges 55 . Ce mouvement d'intensification progressive du contrôle a été si intense que des nombreux auteurs commencent à se référer à un déclin ou même à la mort imminente du modèle de contrôle restreint 56 .

De toute façon, l'idée du modèle de contrôle restreint en tant que « rituel de passage », tendant à avoir un aspect temporaire, rencontre toujours une certaine résistance.

\footnotetext{
51 V. CE Ass. 29 mars 1968, Manufacture française des pneumatiques Michelin, p. 214, AJ 1968, p. 335.

52 CE, 2 nov. 1973, SA Librairie François Maspero, Requête n 82590.

53 CE Sect. 9 juill. 1997, Assoc. Ekin, RFDA 1997.1284, concl. Denis-Linton.

54 CE 29 avril 1994, Cougrand, p. 219, DA 1994, n. 382.

55 Le mouvement exprime le penchant général du droit administratif français vers le renforcement toujours plus grand de la protection juridictionnelle de l'administré. Cela est vérifié dans tous les domaines. Ch. BRÉCHON-MOULĖNES, par exemple, commente la manière par laquelle le juge amdinistratif s'est progressivement senti plus à l'aise pour contrôler les actions administratives en matière économique (v. « La place du juge administratif dans le contentieux économique public », AJDA 2000 p. 679). M. Kalusynzki, pour sa part, commente l'avancée juridictionnelle dans le domaine politique également (v. KALUSYNZKI, Martine. La fonction politique de la Justice: regards historiques. Du souci d'historicité à la pertinence de l'historicisation. In: COMMAILLE, Jacques; KALUSZYNSKI, Martine (Dirs.). La Fonction Politique de la Justice. Paris: Éditions La Découvert, 2007. p. 9-23.

56 Cf. TRUCHET, Didier. Droit Administratif. 3. ed. Paris: PUF, 2010. p. 217.
} 
Sous l'affaire Touzard, en 2006, le commissaire du gouvernement Terry Olson a affronté le thème de manière explicite 57 . II s'agissait de contrôle juridictionnel de l'adéquation entre une sanction disciplinaire appliquée et la faute commise par un agent public matière qui, depuis l'affaire Lebon, en 1978, était soumise au contrôle restreint. Olson examinait s'il était le moment d'abandonner cette jurisprudence et d'avancer vers un contrôle normal. En répondant négativement à cette question - dans ses conclusions qui ont été suivies par le Conseil d'État - il ratifie les raisons par lesquelles il ne faudrait pas transférer la matière en cause vers le contentieux non déférent. En particulier, le commissaire du gouvernement souligne l'existence d'un fort élément politique ou administratif en matière disciplinaire: il comprend des considérations sur quelles seraient la meilleure organisation du service administratif, la meilleure politique de sanction à un moment donné de l'histoire et même des aspects relatifs à l'historique fonctionnel de l'agent public en cause - toutes ces considérations étant alors envisagées comme pouvant être mieux accomplies par l'autorité administrative, plutôt que par les juges 58 .

Ce qui est plus important ici, le commissaire du gouvernement rejette énergiquement l'idée selon laquelle le contrôle restreint aurait vocation à ne constituer qu'un stage temporaire précédant l'évolution jurisprudentielle en direction d'un contrôle plus intense 59 . Olson promeut ainsi une longue et bien fondée défense d'un modèle de contrôle restreint en tant que modèle intermédiaire entre l'absence totale de contrôle et le modèle de contrôle non déférent. À l'instar de l'adéquation de la sanction disciplinaire aux faits qui la motivent, ce modèle de contrôle serait adéquat pour les cas pour lesquels les juges auraient à effectuer une surveillance quelconque, mais sans devoir

57 CE 1er février 2006, Touzard, n² 271676.

58 Selon les mots du commissaire du gouvernement Terry Oıson: « Un autre élément nous semble militer en faveur d'une certaine retenue dans la matière qui vous occupe. Comme on l'a vu, dans le choix de la sanction disciplinaire, l'administration fait entrer en ligne de compte des facteurs qui vont au-delà des manquements fondant la poursuite : il s'agit des antécédents de l'agent et de son attitude générale dans le service. Ces facteurs peuvent conduire à déplacer le curseur, dans le sens de la rigueur ou de la mansuétude. Il en va de même des considérations tirées de l'intérêt général qui s'attache, à un moment donné, à sanctionner tel manquement avec rigueur ou, au contraire, indulgence. Ces deux séries de facteurs nous semblent avoir en commun de ne pas se prêter aisément à une substitution par le juge de son appréciation à celle de l'administration. Ceci est particulièrement vrai des considérations de politique disciplinaire générale qui, à vrai dire, ne concernent pas directement le juge en ce qu'elles renvoient non à un contrôle de légalité mais à des choix de pure opportunité. Le juge de l'excès de pouvoir est-il vraiment apte et légitime pour se placer sur ce terrain ? A notre avis, rien n'est moins sûr » (in CE 1er février 2006, Touzard, n²71676).

59 Le commissaire du gouvernement Terry OLson affirme: «Tout d'abord l'argument selon lequel le contrôle d'erreur manifeste d'appréciation ne serait qu'une sorte de transition, de « sas » entre une absence de contrôle et un entier contrôle ne nous convainc nullement. Le contrôle de l'erreur manifeste d'appréciation est un type de contrôle qui se veut adapté à ce que le juge de l'excès de pouvoir veut et peut contrôler. Or il existe des cas dans lesquels le juge demeure en deçà d'un contrôle même restreint et l'on parle alors parfois d'un contrôle " infra minimum ». Tel est le cas, par exemple, de certains domaines que le juge entend laisser dans un espace absolument discrétionnaire. (...) Il est donc a fortiori logique et justifiable que certains choix faits par l'administration demeurent régis en excès de pouvoir par un contrôle d'erreur manifeste » (in CE 1er février 2006, Touzard, $\left.n^{\circ} 271676\right)$. 
aller jusqu'au point de refaire le raisonnement réalisé par l'administration. Dans ce sens, il serait voué à la pérennité. D'ailleurs, la solution que le commissaire du gouvernement a proposée et qui a été acceptée par le Conseil d'État est toujours en vigueur, dès lors qu'elle a été confirmée lors de décisions prononcées ultérieurement60.

Cet article a voulu promouvoir un examen de la déférence juridictionnelle dans une perspective dynamique. Plutôt que de s'interroger sur la manière par laquelle les modèles de contrôle juridictionnel déférent dans chaque juridiques sont actuellement, l'idée était de s'interroger sur la manière par laquelle ils y sont parvenus, ou comment ont-ils évolué au fil des décennies. Ainsi, ont été analysé le contexte historique et jurisprudentiel de leur création et des aspects relatifs à leur évolution ultérieure.

Cette perspective a révélé des circonstances très intéressantes. La contraposition de l'histoire de la création du modèle de contrôle juridictionnel déférent, avec d'un côté le Canada, les États-Unis et l'Italie, et de l'autre la France, a démontré que la limitation de l'intervention juridictionnelle peut servir à des propos différents. Dans l'hypothèse la plus intuitive, elle implique un mouvement de rétractation juridictionnelle, consistant en l'exhortation de l'auto restriction des juges. Mais, contre-intuitivement, l'initiative peut signifier également un mouvement opposé, dans le sens de l'élargissement de l'intervention juridictionnelle. Ce fût le cas de la France, où les juges administratifs ont fait valoir le modèle de contrôle déférent afin d'introduire une surveillance juridictionnelle minimum dans des domaines qui jusqu'alors échappaient à tout contrôle.

Les différences d'approche peuvent être senties clairement à la lecture des jugements les plus importants. Dans ceux relatifs aux trois premiers systèmes juridiques mentionnés plus haut, l'effort argumentatif vise à convaincre qu'il est nécessaire moins de contrôle. Une bataille doit être menée contre les partisans d'une intervention juridictionnelle plus large - ces sont eux les perdants au final. Au contraire, dans les jugements français qui ont consacré la création du modèle de contrôle déférent, l'effort argumentatif vise à justifier l'apport de plus de contrôle, plus d'intervention juridictionnelle, dans des domaines qui avant leur étaient réfractaires.

L'examen de l'évolution des modèles de contrôle juridictionnel déférent, pour sa part, a démontré que, dans quelques systèmes juridiques, ils se sont trouvés en nette ascension; dans d'autres, en net déclin. Le Canada est le plus grand exemple du premier cas: le modèle de contrôle du caractère raisonnable est aujourd'hui appliqué à la majorité des cas relatifs aux autorités administratives spécialisées. Le modèle non déférent

60 V., par exemple, CE 2 sept. 2009, req. n. 310932, Centre hospitalier Fernand-Langlois ; et CE 12 janv. 2011, req. n. 338461, Matelly. 
de la correction s'applique uniquement à des hypothèses dans lesquelles la décision administrative contrôlée est particulièrement sensible du point de vue juridique.

Aux États-Unis, la situation n'est pas si claire, mais il paraît exister aussi une dissémination de l'orientation déférentielle. En cas de contrôle d'interprétations législatives, la doctrine Skidmore pourra s'appliquer, même à défaut d'application de la doctrine Chevron - toutes les deux étant de nature déférentielle. En dehors du champ des interprétations législatives, les hypothèses d'application du contrôle non déférent (sous le modèle de novo) sont très rares au sein du règlement générique de l'Administrative Procedure Act.

La situation contraire peut être vérifiée dans les systèmes juridiques étudiés en Europe. En Italie, l'un des modèles déférents de contrôle, appelé contrôle faible, n'a duré que trois ans et a été abandonné à la suite de fortes critiques mettant en cause son inefficience juridictionnelle. En France, après avoir été disséminé dans des domaines qui préalablement échappaient à tout contrôle juridictionnel, le contrôle restreint a cédé progressivement de la place en faveur du contrôle non déférent, dit « normal ». La situation a conduit quelques auteurs à décréter la « mort imminente » du modèle de contrôle restreint, tandis que d'autres ont souligné sa prétendue nature transitoire.

Cela paraît constituer une nouvelle évidence de la juridictionnalisation forte et croissante du droit administratif français. Même dans hypothèses de plus en plus rares d'application du contrôle juridictionnel restreint, il pourrait ne pas refléter proprement une attitude respectueuse des juges vis-à-vis de l'administration publique, dans des matières qui lui sont réservées. Au lieu de cela, une telle application du contrôle restreint pourrait indiquer une sorte de mouvement incomplet d'intervention. C'est l'idée propagée par certains auteurs, selon laquelle le contrôle restreint en France consisterait en un simple « rituel de passage » en direction d'un contrôle plus intense.

Cette proposition est mise en cause par le fait que quelques matières résistent encore au passage vers un contrôle normal - et d'autres échappent toujours à tout contrôle. Dans ce sens, l'article a présenté aussi les conclusions rendues par le commissaire du gouvernement Terry Olson sous l'affaire Touzard, dans lesquelles il a soutenu le maintien d'une alternative intermédiaire entre le contrôle normal et l'absence de contrôle, en fournissant notamment des raisons institutionnelles, selon lesquelles les juges devaient se tenir à distance de certaines matières plus appropriées au corps administratif. 


\section{RÉFÉRENCES}

BONNARD, Roger. Le Contrôle Juridictionnel de l'Administration: Étude de Droit Administratif Comparé. Paris: Dalloz, 2005.

BRÉCHON-MOULÈNES Christine. La place du juge administratif dans le contentieux économique public. Actualité juridique - Droit Administratif, Paris, v. 56, n. 9, p. 679-686, sept. 2000.

CRAIG, Jared. Defending city hall after Dunsmuir. Alberta Law Review Edmonton, v. 46, n. 1, p. 275-297, nov. 2008.

DYZENHAUS, David. The politics of deference: judicial review and democracy. In: TAGGART, Michael (ed.). The Province of Administrative Law. Oxford: Hart Publishing, 1997.

DYZENHAUS, David. The logic of the rule of law: lessons from Willis. The University of Toronto Law Journal, Toronto, v. 55, n. 3, p. 691-714, jun./sept. 2005.

DYZENHAUS, David; FOX-DECENT, Evan. Rethinking the process/substance distinction: Baker versus Canada. The University of Toronto Law Journal, Toronto, v. 51, n. 3, p. 193-242, jun./sept. 2001.

ESSENS, Oda; GERBRANDY, Anna; LAVRIJSSEN, Saskia (Ed.). National Courts and the Standard of Review in Competition Law and Economic Regulation. Groningen: Europa Law Publishing, 2009.

GARRY, Patrick M. Judicial review and the 'hard-look' doctrine. The Nevada Law Journal, Las Vegas, v. 7, n. 1, p. 151-170, sept./dec. 2006.

JACOBS, Laverne. Developments in administrative law: the 2007-2008 term - The impact of Dunsmuir. Supreme Court Law Review, Ottawa, v. 43, n. 2, p. 1-34, dec. 2008.

JAFFE, Louis L. The right to judicial review I. Harvard Law Review, New Haven, v. 71, n. 3, p. 401-437.

KALUSYNZKI, Martine. La fonction politique de la Justice: regards historiques. Du souci d'historicité à la pertinence de l'historicisation. In: COMMAILLE, Jacques; KALUSZYNSKI, Martine (Dirs.). La Fonction Politique de la Justice. Paris: Éditions La Découvert, 2007.

LANGILLE, Brian. Judicial review, judicial revisionism and judicial responsibility. Revue Générale de Droit, Ottawa, v. 17, n. 1-2, p. 169-192, 1986.

LI, Zhang. Le Contrôle Juridictionnel de la Légalité des Actes Administratifs en Chine: Eléments d'Analyse Comparée des Contentieux Administratifs Chinois et Français. Bruxelles: Bruylant, 2010.

MACKLIN, Audrey. Standard of review: the pragmatic and functional test. In: FLOOD, Coleen; SOSSIN, Lorne (Eds.). Administrative Law in Context. Toronto, Emond Montgomery, 2008. 
MARCHETTI, Barbara. Pubblica Amministrazione e Corti negli Stati Uniti: il Judicial Review sulle Administrative Agencies. Padova: CEDAM, 2005.

MERRILL, Thomas W. Judicial deference to Executive precedent. The Yale Law Journal, New Haven, v. 101, n. 5, p. 969-1041, mar. 1992.

MERRILL, Thomas W. The story of Chevron: the making of an accidental landmark. In: STRAUSS, Peter (Ed.). Administrative Law Stories. New York: Thomsom/West, 2006.

MULLAN, David J. Recent developments in administrative law - The apparent triumph of deference! Canadian Journal of Administrative Law and Practice, v. 12, n. 2, p. 191-, 1998.

ODENT, Raymond. Contentieux Administratif. Paris: Institut d'Études Politiques de Paris, 1971.

RISK, Richard C. B. In Memoriam: John Willis. The University of Toronto Law Journal, Toronto, v. 47, p. 301-304, 1997.

SUNSTEIN, Cass R. Deregulation and the hard-look doctrine. The Supreme Court Review, Chicago, v. 24, p. 177-213, 1983.

SUNSTEIN, Cass R. Law and administration after Chevron. Columbia Law Review, New York, v. 90, n. 8, p. 2071-2120, dec. 1990.

TRUCHET, Didier. Droit Administratif. 3. ed. Paris: PUF, 2010.

WERHAN, Keith. The neoclassical revival in administrative law. Administrative Law Review, Washington, v. 44, n. 3, p. 567-628, dec.1992.

WILLIS, John. Three approaches to administrative law: the judicial, the conceptual and the functional. The University of Toronto Law Journal, Toronto, v. 1, p. 53-81, 1935.

WOOLHANDLER, Ann. Judicial deference to administrative action - A revisionist history. Administrative Law Review, Washington, v. 43, p. 197-223, 1991. 